\title{
Consumo de álcool e outras substâncias psicoativas entre universitários e a prática de binge drinking
}

\author{
Submetido - 17 dez. $2020 \quad$ Aprovado - 02 abr. $2021 \quad$ Publicado - 01 maio 2021
}

\section{http://dx.doi.org/10.52755/sas.v2i2.105}

André Tomaz Terra Júnior

Doutor em Medicina (Clínica Cirúrgica) pela Faculdade de Medicina de Ribeirão Preto/Universidade de São Paulo (USP). Docente e Coordenador do Curso de Farmácia da FANORTE (Instituição de Ensino Superior de Cacoal-RO).E-mail: andretomaz@alumni.usp.br.

Jéssica Castro dos Santos

Mestre em Saúde e Educação pela Faculdade de Ribeirão Preto - UNAERP. Especialista em Terapia Intensiva pela Faculdade Inspirar. Coordenadora e Docente do curso de Bacharelado em Fisioterapia da Faculdade de Educação e Meio Ambiente - FAEMA. E-mail: jessica_castro08@hotmail.com.

Leandro Fantin de Pontes

Bacharel em Farmácia pela Faculdade de Educação e Meio Ambiente - FAEMA. Email: leandrofp89@gmail.com.

Dione Rodrigues Fernandes Bacharel em Farmácia pela Faculdade de Educação e Meio Ambiente - FAEMA. E-mail: dionefernandes.claro@gmail.com.

Licenciado em Educação Física - Faculdade de Educação e Meio Ambiente - FAEMA. Email:diogoadmr@gmail.com.

\section{RESUMO}

O álcool está entre as substâncias psicoativas mais consumidas entre os jovens universitários, representando um sério problema de saúde pública. A modalidade do consumo de álcool "Binge Drinking" é caracterizada pelo consumo em grande quantidade da substância em uma mesma ocasião. Diante do exposto, este estudo teve como objetivo verificar a prevalência e o padrão de consumo de álcool entre os acadêmicos de uma instituição particular de ensino superior situada no interior do estado de Rondônia. Adotou-se a metodologia de estudo transversal em que foi utilizada uma amostra de conveniência não probabilística. Aplicou-se um questionário estruturado com questões validadas para autopreenchimento. $A$ associação entre variáveis foi aferida pelo teste qui-quadrado, assumindo-se o nível de significância de $5 \%$ ( $p<0,05)$. Foram analisados 525 questionários. Dentre os resultados encontrados nota-se que 46,1\% dos acadêmicos consomem abusivamente bebidas alcoólicas, 38,2 \% haviam bebido de forma agressiva e maciça pelo menos uma vez ao mês e 54,4\% já haviam consumido bebidas alcoólicas antes de ingressar no ensino superior. Comparativamente aos homens, as mulheres representam o maior índice para consumo e consumo mais nocivo de álcool (69\%). Na relação entre consumo de álcool e comportamento de risco, $58,3 \%$ dos universitários declararam que não usam preservativos nas relações sexuais, 13,7\% tinham ingerido bebida alcoólica antes da relação sexual e $53,1 \%$ já tiveram mais de três parceiros sexuais. Em consonância com outros achados na literatura científica, este estudo indica a relação da prática de Binge Drinking com o aumento das chances de manifestação de problemas relacionados ao uso de álcool e a outros comportamentos de risco, principalmente entre acadêmicas. As diferenças do padrão de consumo entre os gêneros podem ser importantes em ações de prevenção mais eficazes sobre o uso de álcool entre os universitários.

Palavras-chave: Binge Drinking; Alcoolismo; Intoxicação Alcoólica.

Este é um trabalho de acesso aberto e distribuído sob os Termos da Creative Commons Attribution Attribution-NonCommercial-ShareAlike 4.0 International. 


\title{
Consumption of alcohol and other psychoactive substances between university students and the practice of binge drinking
}

\begin{abstract}
Alcohol is among the most consumed psychoactive substances by higher education students, representing a serious public health problem. "Binge Drinking" is a category characterized by the consumption of a large quantity of alcohol on a single occasion. In view of the above stated, this study aimed to verify the prevalence and pattern of alcohol consumption by students of a private higher education institution located in the state of Rondônia countryside, Brazil. The cross-sectional study methodology was adopted utilizing a non-probabilistic convenience sample. A structured questionnaire was applied with validated questions for self-completion. The association between variables was assessed using the chi-square test, assuming a significance level of $5 \%(p<0.05) .525$ questionnaires were analyzed. Among the results found, it is noted that $46.1 \%$ of academics abuse alcoholic beverages, $38.2 \%$ had drunk aggressively and massively at least once a month and $54.4 \%$ had already consumed alcoholic beverages before joining higher education. Compared to men, women represent the highest rate for consumption and most harmful consumption of alcohol (69\%). Comparing alcohol consumption and risky behavior, 58.3\% of university students declared that they did not use condoms during sexual intercourse, $13.7 \%$ had drunk alcohol before sexual intercourse and $53.1 \%$ had already had more than three sexual partners. In accordance with other findings in the scientific literature, this study indicates the relationship between the practice of Binge Drinking and the increased chances of manifesting problems related to the use of alcohol and other risky behaviors, especially among females. Differences in the pattern of consumption between genders can be important in more effective prevention actions on alcohol use among university students.
\end{abstract}

Keywords: Binge Drinking; Alcoholism; Alcoholic Intoxication.

\section{Consumo de alcohol y otras sustancias psicoactivas entre estudiantes universitarios y práctica de binge drinking}

\section{RESUMEN}

El alcohol se encuentra entre las sustancias psicoactivas más consumidas entre los estudiantes universitarios, lo que representa un grave problema de salud pública. La modalidad de consumo de alcohol "Binge Drinking" se caracteriza por el consumo de una gran cantidad de la sustancia en la misma ocasión. Dado lo anterior, este estudio tuvo como objetivo verificar la prevalencia y patrón de consumo de alcohol entre estudiantes de una institución privada de educación superior ubicada en el interior del estado de Rondônia, Brasil. Se adoptó la metodología de estudio transversal en la que se utilizó una muestra de conveniencia no probabilística. Se aplicó un cuestionario estructurado con preguntas validadas para autocompletar. La asociación entre variables se evaluó mediante la prueba de chi-cuadrado, asumiendo un nivel de significancia del $5 \%(p<0,05)$. Se analizaron 525 cuestionarios. Dentre os resultados encontrados nota-se que $46,1 \%$ dos acadêmicos consomem abusivamente bebidas alcoólicas, $38,2 \%$ haviam bebido de forma agressiva e maciça pelo menos uma vez ao mês e 54,4\% já haviam consumido bebidas alcoólicas antes de ingressar no enseñanza superior. En comparación con los hombres, las mujeres representan la tasa más alta de consumo y el consumo más nocivo de alcohol (69\%). En la relación entre el consumo de alcohol y las conductas de riesgo, el 58,3\% de los estudiantes universitarios declararon no utilizar preservativo durante las relaciones sexuales, el 13,7\% había bebido alcohol antes de la relación sexual y el $53,1 \%$ ya había tenido más de tres parejas sexuales. En línea con otros hallazgos de la literatura científica, este estudio indica la relación entre la práctica del consumo excesivo de alcohol y las mayores posibilidades de manifestar problemas relacionados con el consumo de alcohol y otras conductas de riesgo, especialmente entre los académicos. Las diferencias en el patrón de consumo entre géneros pueden ser importantes para acciones de prevención más efectivas sobre el consumo de alcohol entre los estudiantes universitarios.

Palabras clave: Binge Drinking; Alcoholismo; Intoxicación Alcohólica. 


\section{Introdução}

A adolescência é um período marcado pela transição entre a puberdade e a maturidade da fase adulta, sendo caracterizada por profundas modificações de ordem fisiológica, psicológica, sociais e outros aspectos relevantes que se distinguem de outras etapas da vida. É uma fase também de experimentar novas sensações e experiências, podendo ser considerado um período de alto risco com relação ao uso de álcool, drogas e outras substâncias psicoativas (BOCHNER, 2006; WHO, 2014).

Apesar de, no Brasil, a venda de bebidas alcoólicas ser proibida para menores de 18 anos, o consumo de álcool pelos jovens menores de idade ainda é comum (MALTA et al., 2011). Conforme a WHO (2010), o consumo dessas substâncias é globalmente alarmante e seu uso abusivo é um fator crítico para a saúde pública em diversos países, notadamente nos países em desenvolvimento como o Brasil.

Destaca-se que o consumo abusivo do álcool se constitui como agravante de diversas doenças, além de ser registrado globalmente como a terceira principal causa de incapacidades e de mortes prematuras em um índice aproximado de 2,5 milhões de indivíduos por ano. Assim informa documento da Organização Mundial de Saúde intitulado "Estratégia Global para Reduzir os Efeitos Nocivos da Utilização do Álcool”, de 2010, indicando a faixa etária de 15 a 29 como a principal envolvida nas estatísticas (WHO, 2010).

Estudos epidemiológicos mostram uma alta prevalência de consumo de substâncias psicoativas na faixa etária de 18 a 25 anos entre os jovens brasileiros. Sendo uma faixa etária onde há um aumento significativo de jovens ingressando no ensino superior, isso os torna alvo de pesquisas sobre o tema (GALDUROZ et al., 2005; JOHNSTON et al., 2006).

Em um levantamento feito por Fiorini (2003), relata-se que os jovens consultados consumiam álcool antes de ingressarem no nível superior. Esse dado vai ao encontro com os dados do $1^{\circ}$ Levantamento Nacional sobre os padrões de consumo de álcool na população brasileira em 2007 
(LARANJEIRAS et al., 2005), que avaliou o padrão de consumo de álcool entre os jovens brasileiros, no qual os jovens relataram que tiveram o primeiro contato com essa substância entre 14,8 e 17,3 anos.

É importante ressaltar que não há uma relação entre ensino superior e consumo de álcool e substâncias psicoativas e, geralmente, o uso de substâncias psicoativas ocorre inicialmente na adolescência. Esse fato parece tornar factível que na universidade possam existir fatores que potencializem o uso dessas substâncias psicoativas. (TAVARES; BÉRIA; LIMA, 2001).

O Álcool está entre as substâncias psicoativas mais consumidas no mundo, principalmente entres os jovens universitários (WHO, 2014), e esse consumo abusivo apresenta um aumento expressivo e consiste em um problema de saúde pública, mas atualmente está assumindo uma importância crescente como causa de doença, incapacidade e morte prematura em todo o mundo (PUIG-NOLASCO et al., 2011). Ademais, esse consumo abusivo entre os universitários é cada vez mais frequente, sendo considerada a população que apresenta o maior padrão de consumo (SANCHEZ, 2015).

Os universitários da área da saúde, principalmente, estão entre os jovens comumente vulneráveis aos episódios de Binge Drinking (BD), termo empregado para determinar o uso excessivo e episódico de álcool (ROCHA et al., 2011). São considerados BD a ingestão abusiva de bebidas alcoólicas em uma única ocasião, sendo de cinco ou mais doses por homens ou quatro ou mais por mulheres, desconsiderando a frequência de uso (CARDOSO et al.,2015).

Alguns autores tentam esclarecer esse comportamento abusivo de BD entre os jovens, principalmente entre os universitários da área da saúde, embora essa população acadêmica conheçam bem os efeitos prejudiciais decorrentes do uso abusivo de bebidas alcoólicas, a busca por substâncias capazes de aliviar situações de intenso estresse e ansiedade do seu cotidiano, fazendo com que resulte na busca ocasional pelo uso e abuso de 
bebidas alcoólicas como simplesmente um refúgio, porém essas atitudes seguramente podem gerar problemas pessoais, profissionais e sociais. (NUNES et al., 2012).

Essa prática é considerada um comportamento de risco, que pode provocar alterações fisiológicas e, possivelmente, levar a quadros de intoxicação, coma e, em situações extremas, é passível de causar óbito. Outros fatores também podem ser associados a essa prática, como baixo desemprenho escolar, acidentes de trânsito, violência e o risco de desenvolver alcoolismo no futuro (SANCHEZ, 2015).

Há uma variabilidade farmacocinética entre os gêneros, na qual as mulheres se apresentam mais vulneráveis e mais responsivas à ação do álcool quando ingerido quantidades equivalentes, devido ao menor peso e maior proporção de gordura corporal, além de menor capacidade de metabolismo hepático do álcool (BOYLE et al., 2013).

Além de tudo, Pechansky et al. (2004) mencionam os efeitos maléficos do álcool na neuroquímica cerebral de adolescentes, podendo causar "[...] pior ajustamento social e no retardo do desenvolvimento de suas habilidades, já que um adolescente ainda está se estruturando em termos biológicos, sociais, pessoais e emocionais.". Vale ressaltar que dentre das diversas consequências do uso do uso abusivo do álcool pode favorecer condutas comportamentais inadequadas, como a associação com outros tipos drogas e comportamentos sexual de risco, aumentado a chance de contrair DST (REIS; OLIVEIRA, 2015; TSUDA; CHRISTOFF, 2017; NUNES et al.,2012).

O objetivo desta pesquisa foi verificar a prevalência e o padrão de consumo alcoólico e fatores associados ao "Binge drinking" em acadêmicos matriculados em cursos de graduação de uma instituição particular de ensino superior situada no interior do estado de Rondônia. 


\section{Material e Métodos}

A pesquisa se trata de um estudo transversal em que será utilizada uma amostra de conveniência não probabilística de estudantes universitários com matrícula regular em Cursos de Graduação de uma instituição particular de ensino superior do interior de Rondônia.

O presente estudo foi aprovado pelo Comitê de Ética em Pesquisa, constituído nos termos da Resolução 196/96, do Conselho Nacional de Saúde, do Ministério da Saúde (CNS/MS) sob o parecer №: 2.310 .781 (CAAE 64340517.9.0000.5601). O Termo de Consentimento Livre e Esclarecido (TCLE) foi disponibilizado na página inicial do instrumento online.

Do total de 685 estudantes matriculados anualmente, por meio do cálculo amostral, aceitando-se um erro de estimação não superior a $2 \% \mathrm{e}$ um nível de confiança de 95\%; e adotando-se procedimento de amostragem sistemática, foi estimado entrevistar 550 estudantes, para prevenir possíveis perdas.

Foram incluídos estudantes universitários devidamente matriculados nos cursos de Graduação em Farmácia, Enfermagem, Fisioterapia, Psicologia, Agronomia, Engenharia Ambiental e Sanitária, maiores de 18 anos de idade, de ambos os sexos, que aceitaram o preenchimento online do questionário para coleta de dados. Aqueles estudantes universitários que se negaram a responder o questionário online da pesquisa ou deixaram questionários respondidos incompletos não foram considerados elegíveis. Para coleta de dados foi utilizado um questionário online composto por informações sociodemográficas, o Youth Risk Behavior Surveillance System (YRBSS) e o Alcohol, Smoking and Substance Involving Screening Test (ASSIST). Por meio de um questionário online foi identificado o padrão de consumo de bebidas alcoólicas entre jovens universitários e outras associações.

Os dados foram analisados com o auxílio do programa Statistical Package for the Social Science (SPSS 17). Utilizou-se ferramentas 
estatísticas como análise univariada para a descrição das variáveis quantitativas relacionadas ao perfil socioeconômico e a análise bivariada para verificar a associação entre o uso de bebidas alcoólicas e as variáveis independentes (sexo, idade, religião e nível socioeconômico) por meio do teste Qui-quadrado com nível de significância de 5\%.

\section{Resultados e discussão}

A amostra deste estudo foi composta por 518 universitários matriculados nos cursos de graduação em uma instituição particular de ensino superior de Rondônia, sendo divididas em dois grandes grupos: área da saúde $(70,9 \%, n=367)$ e áreas de exatas, humanas e ciências da natureza $(29,2 \%, n=151)$. Dos cursos de graduação que participaram da pesquisa (Tabela 1), 36,9\% dos entrevistados estão matriculados no curso de Farmácia e 16,21\% no curso de Fisioterapia, 17,8\% no Curso de Enfermagem e $29,2 \%$ nos demais cursos.

Tabela 1. Caracterização da amostra total quanto ao curso de graduação.

\begin{tabular}{lc}
\hline Curso de Graduação & Amostra Total - N (\%) \\
\hline Farmácia & $191(36,9)$ \\
Fisioterapia & $84(16,2)$ \\
Enfermagem & $92(17,8)$ \\
Demais cursos & $151(29,2)$ \\
\hline
\end{tabular}

*Demais cursos (Administração, Agronomia, Ciências Contábeis, Licenciatura em Química, Licenciatura em Física, Engenharia Civil).

Os estudantes apresentaram uma média de idade de 23,12 anos, obtidos pela média aritmética de intervalos de idades $\left(X=\sum\left(X A^{*} X M\right) / \sum X A\right)$, que foram divididos em quatro grupos de faixa etária com idade mínima de 18 anos e acima de 35 anos, onde 367 (70,7\%) dos universitários têm entre 1823 anos, $82(15,8 \%)$ têm idade entre 24 - 29 anos, $45(8,9 \%)$ têm de 30 -35 anos e 24 (4,61\%) têm idade acima de 35 anos.

Quando questionados sobre o uso concomitante de álcool durante a vida com MTF e outras substâncias psicoativas apresentaram uma relação 
moderada a fraca entre os universitários (Tabela 2), foi realizado uma regressão múltipla na procura encontrar uma combinação linear de previsores (Ritalina na vida, Tabaco, Tranquilizantes, H/S, Anfetamina, Maconha e Fluoxetina) que se correlacionam de forma máxima com a variável de saída (Álcool na vida). O modelo conseguiu explicar 14\% da relação existente entre o consumo de álcool na vida e os previsores supracitados, não havendo colinearidade. Como os previsores Tabaco e maconha são variáveis binárias (sim ou não) e álcool na vida foi medido em intervalos de dias, o fato de os alunos consumirem tabaco aumenta em 1,56 o intervalo de dias de consumo de álcool na vida e 1,23 o intervalo de dias de consumo de álcool se consumir Maconha.

Tabela 2. Consumo de bebida alcoólica durante a vida e a relação com o uso de Ritalina e outras Substâncias Psicoativas.

\begin{tabular}{ccccc}
\hline & \multicolumn{3}{c}{ Regressão múltipla } \\
\hline Variáveis & $\mathrm{R}^{2}=$ & $\mathrm{F}=$ & Var. significativas & \\
\hline $\begin{array}{c}\text { Consumo de álcool } \\
\mathbf{x}\end{array}$ & & & & Tabaco $(\mathrm{b}=1,558$, \\
$\begin{array}{c}\text { Ritalina e outras } \\
\text { Substâncias Psicoativas }\end{array}$ & 0,142 & 12,074 & Tabaco e maconha & $\begin{array}{c}\text { Beta }=0,272) \text { Maconha } \\
(b=1,285, \text { Beta }=0,169)\end{array}$ \\
\hline
\end{tabular}

Outro ponto importante dessa pesquisa foi fazer uma avaliação de atitudes e comportamentos de risco ligados ao uso de álcool e outras drogas (Tabela 03), onde o modelo conseguiu explicar, através de uma regressão logística, cruzando as variáveis "uso de álcool”, "álcool na vida" e "drogas e comportamentos de risco", que há uma probabilidade associada à ocorrência de $54,6 \%(R 2=56,4)$ entre apenas o uso de álcool na vida foi significativo, ou seja, a chances do aluno adotar um comportamento de risco é maior à medida que o intervalo de dias de consumo de álcool aumenta.

Tabela 3. Inter-relação do uso de álcool, álcool na vida e drogas e comportamentos de risco.

\begin{tabular}{cccc}
\hline \multirow{2}{*}{ Variáveis } & \multicolumn{2}{c}{ Regressão Logística } \\
\cline { 2 - 4 } & $\mathrm{R}^{2}$ & $\begin{array}{c}\text { Var. } \\
\text { significativas }\end{array}$ & Valores \\
\hline $\begin{array}{c}\mathbf{X} \\
\text { Uso de álcool e drogas }\end{array}$ & $54,6 \%$ & Álcool na vida & $\mathrm{b}=0,107$, \\
EXP: 1,112
\end{tabular}


Esse modelo servirá como parâmetro para uma maior compreensão e entendimento desses universitários, possibilitando adequações de programas preventivos de educação em saúde, auxiliando na melhoria de qualidade de vida e medidas de controle. Como consequências do consumo de drogas entre estudantes universitários, podemos citar: acidentes automobilísticos, violência, comportamento sexual de risco, prejuízos acadêmicos, diminuição da percepção e estresse (MENDONÇA, et al., 2018; REIS; OLIVEIRA, 2015; NUNES, et al. 2012; WAGNER, ANDRADE, 2008; PILLON et al., 2005).

Em uma análise de gêneros (Tabela 4), detectamos que as mulheres $(69 \%, n=165)$, representam o grupo populacional de risco para consumo de álcool e que estão bebendo de forma mais nociva em relação aos homens $(31 \%, n=74)$. Esses dados estão de acordo com o II Levantamento Nacional de Álcool e Drogas - LENAD (LARANJEIRAS et al.,2012) além de outros dados nacionais e internacionais, que abordam elevadas prevalências do padrão de consumo BD entre estudantes universitários do sexo feminino (TAVOLACCI et al.,2016; FACHIN; FURTADO, 2013; ANDRADE et al., 2010), considerando-se que esses dados são agravantes, há uma maior vulnerabilidade do sexo feminino em relação ao $\mathrm{BD}$ e ao consumo excessivo de bebidas alcoólicas, tornando-se necessário uma conscientização dessa população através de campanhas educativas que abordem o uso inadequado e abusivo do álcool e seus efeitos prejudiciais ao organismo.

A compreensão dessas diferenças entre gêneros e de sues fatores subjacentes ao consumo de álcool podem contribuir para a elaboração de ações preventivas mais específicas e eficazes sobre o consumo de álcool em função do sexo (FACHINI; FURTADO, 2013).

Ainda acompanhando a Tabela 4, 54,4\% ( $n=282)$ dos alunos pesquisados afirmaram que tiveram um primeiro contato com bebidas alcóolicas com idade inferior a 17 anos. Esse comportamento precoce pode ser considerado um fator de exposição para desenvolvimento de problemas 
de saúde na idade adulta, além de ser um fator de predisposição para consumo crônico ao longo da vida (STRAUCH et al., 2009).

Tabela 4. Caracterização da amostra quanto à prática de BD.

\begin{tabular}{|c|c|c|c|c|c|}
\hline \multicolumn{6}{|c|}{ Binge drinking ( $\geq 5$ doses) } \\
\hline \multirow[t]{2}{*}{ Variáveis } & \multicolumn{2}{|c|}{ Sim } & \multicolumn{2}{|c|}{ Não } & \multirow[t]{2}{*}{$\mathbf{P}$} \\
\hline & (n) & $(\%)$ & (n) & $(\%)$ & \\
\hline \multicolumn{6}{|l|}{ Sexo } \\
\hline Feminino & 165 & 69,0 & 190 & 68,1 & 0,819 \\
\hline Masculino & 74 & 31,0 & 89 & 31,9 & \\
\hline \multicolumn{6}{|l|}{ Idade } \\
\hline $18-23$ & 175 & 73,2 & 191 & 68,5 & 0,318 \\
\hline $24-29$ & 35 & 14,6 & 47 & 16,8 & \\
\hline $30-35$ & 22 & 9,2 & 24 & 8,6 & \\
\hline Acima de 35 & 7 & 2,9 & 17 & 6,1 & \\
\hline \multicolumn{6}{|c|}{ Período do curso } \\
\hline $1^{\circ}-3^{\circ}$ & 112 & 46,9 & 125 & 44,8 & 0,754 \\
\hline $4^{\circ}-6^{\circ}$ & 79 & 33,1 & 101 & 36,2 & \\
\hline $7^{\circ}-9^{\circ}$ & 48 & 20,1 & 53 & 19,0 & \\
\hline \multicolumn{6}{|l|}{ Religião } \\
\hline Católica & 100 & 41,8 & 112 & 40,1 & 0,766 \\
\hline Nenhuma & 6 & 2,5 & 5 & 1,8 & \\
\hline Outras & 133 & 55,6 & 162 & 58,1 & \\
\hline \multicolumn{6}{|l|}{ Moradia } \\
\hline Família & 208 & 74,6 & 165 & 69,0 & 0,163 \\
\hline Outros & 71 & 25,4 & 74 & 31,0 & \\
\hline
\end{tabular}

A prevalência do consumo de álcool pelos estudantes pelo menos uma vez na vida foi de $80,97 \%$ ( $n=419)$, semelhante ao que observado por Cardoso et al. (2015) e Puig-Nolasco (2011), onde essa prevalência foi de $74,9 \%$ e $71,9 \%$, respectivamente. Estes dados mostram o elevado consumo alcoólico entre jovens estudantes em ambos os sexos, principalmente entre os universitários da área da saúde. Esses universitários foram divididos em grupos de faixa etária, na intensão de determinar qual faixa etária tem o consumo maciço de álcool, podemos notar que 73,2\% ( $n=175)$ tem idade de 18 a 23 anos e que $14,6 \%(n=35)$ têm entre 24 a 29 anos, porém quando questionados sobre o primeiro contato com bebidas alcoólicas, $54,4 \%$ ( $n=282)$ já haviam consumido bebidas alcoólicas antes de ingressar na faculdade, esse fato se torna preocupante e nos chama atenção pela necessidade da implantação de políticas públicas em relação a essa temática e também nos remete a importância de intervenções preventivas, educativas e efetivas, através de atividades de promoção da saúde. Alguns autores defendem que essas ações devem ser inseridas ainda no ensino 
fundamental e médio, a fim de minimizar o percentual de consumo e de uso abusivo de bebidas alcoólicas em universitário (NUNES et al., 2012).

Conforme o Gráfico 1, avaliou-se o uso de substâncias psicoativas ao longo da vida, no último ano e no último mês, descrevendo a distribuição do uso de substâncias psicoativas ao longo da vida. Observa-se que o álcool é a substância psicoativa com maiores percentuais de uso ao longo da vida na amostra 74,3\% ( $n=385)$, seguido de Tranquilizantes 23\% $(n=119)$ e Tabaco 19,5\% ( $n=101)$, comparativamente ao uso abusivo de medicamentos e drogas ilícitas. Estes dados corroboram com Andrade et al. (2010) e CEBRID (2010), que elencam o álcool e o tabaco como drogas de maior consumo entre os adolescentes.

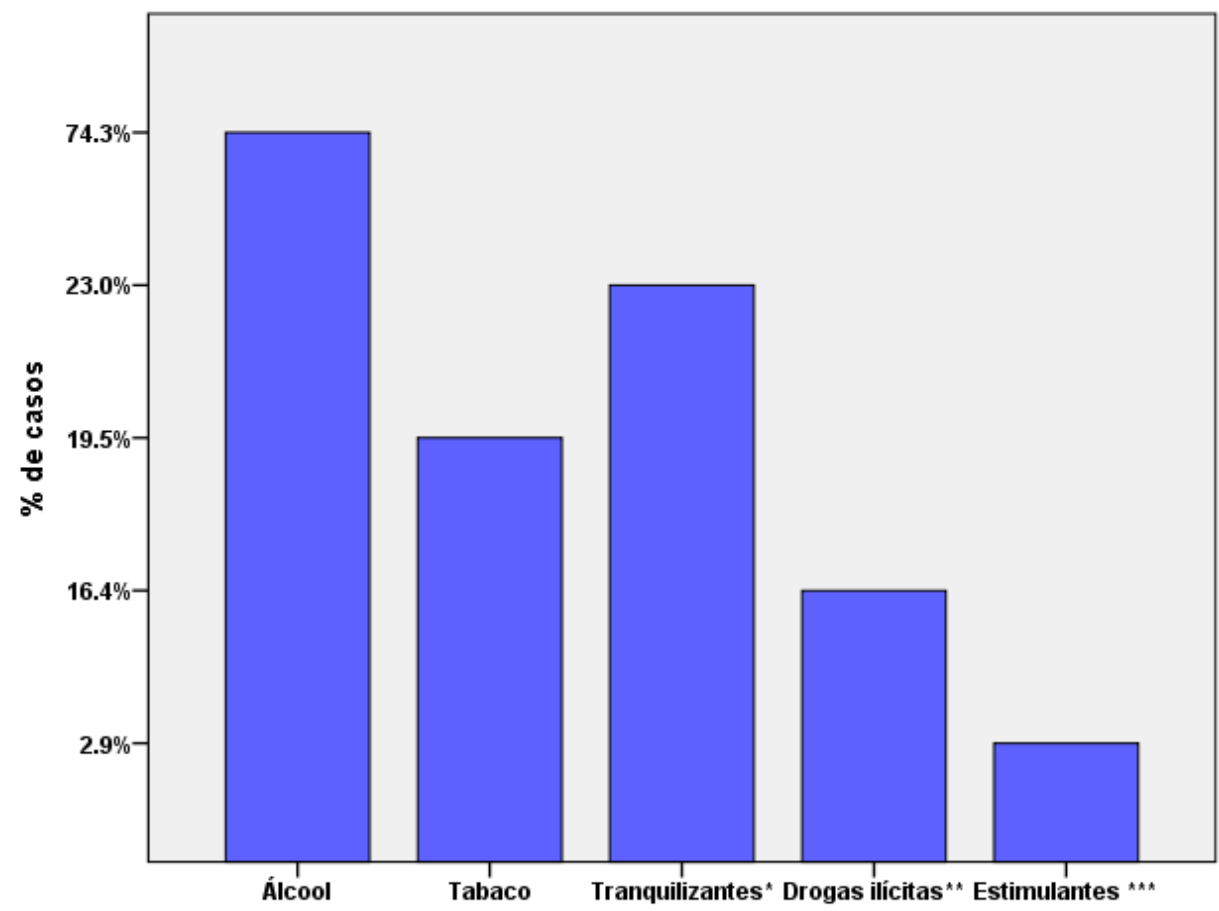

Gráfico 1. Caracterização da amostra quanto ao uso de substâncias

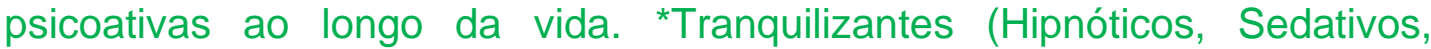
Antidepressivos), ${ }^{* *}$ Drogas ilícitas (Maconha, Crack, Cocaína), ${ }^{* * *}$ Estimulantes (Anfetaminas, Exctasy).

O álcool é a principal droga de consumo entre os adolescentes universitários, devido à facilidade de acesso e preço, e revelou ser um dado preocupante, em relação ao uso de bebidas alcoólicas ao longo da vida, $22,2 \%(n=115)$ dos universitários afirmaram que já beberam mais de 100 dias durante a sua vida, e 58,7\% ( $n=304)$ afirmam que já experimentaram 
em algum momento na sua vida, e apenas $19.1 \%(n=99)$ declaram que nunca experimentaram bebidas alcoólicas durante a vida, como mostra o Gráfico 2. No que nos diz respeito ao abuso de álcool e fatores que podem estar associados a este uso, observamos que possa haver variáveis associadas ao consumo abusivo que não foram listadas.

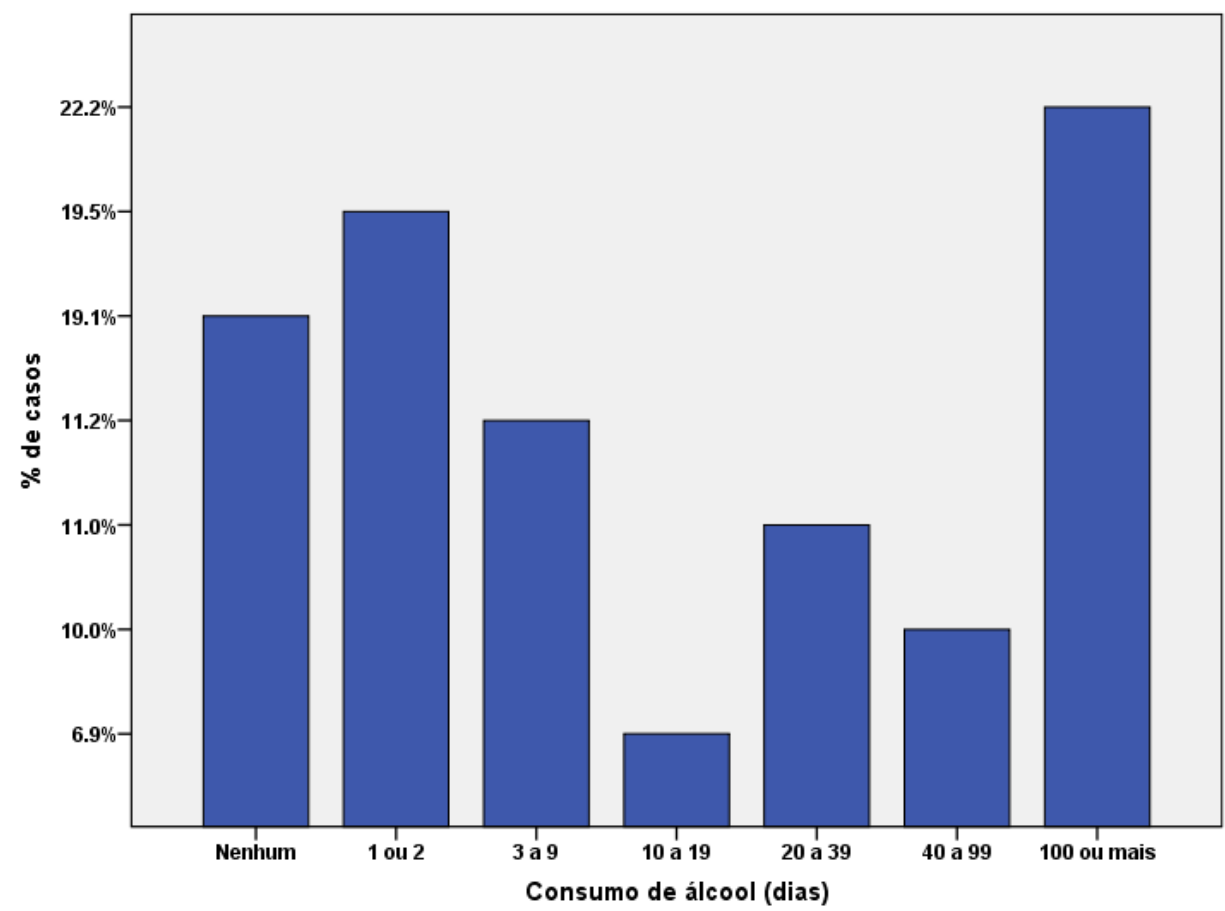

Gráfico 2. Consumo de bebida alcoólica durante a vida (dias).

Quando questionados sobre a frequência com que ingerem bebidas alcoólicas em BD (Gráfico 3), constamos que 23,6\% ( $n=122)$ consome menos de uma vez por mês e $14,9 \%(n=77)$ consome pelo menos uma vez ao mês, tornando essa realidade muito mais preocupante, devido às consequências negativas à saúde física e mental dos jovens e à sociedade em todos os aspectos - econômicos, sociais e emocionais inerentes a acidentes e agravos motivados pelo BD e pela possibilidade de danos para a saúde a longo prazo (CARDOSO et al., 2015; ROCHA et al., 2011).

De acordo com a prevalência nos últimos 30 dias sobre o consumo de BD (Gráfico 4), constata-se que 38,2 \% $(n=198)$ haviam bebido de forma agressiva e maciça pelo menos uma vez ao mês, essa prevalência de ingerir altas quantidade de bebidas alcoólicas de maneira episódica também se mostrou dentro das margens do I Levantamento Nacional sobre o Uso de 
Álcool entre Universitários, que identificou percentual variando de $29,0 \%$ a 43,7\% (CARDOSO et al., 2010).

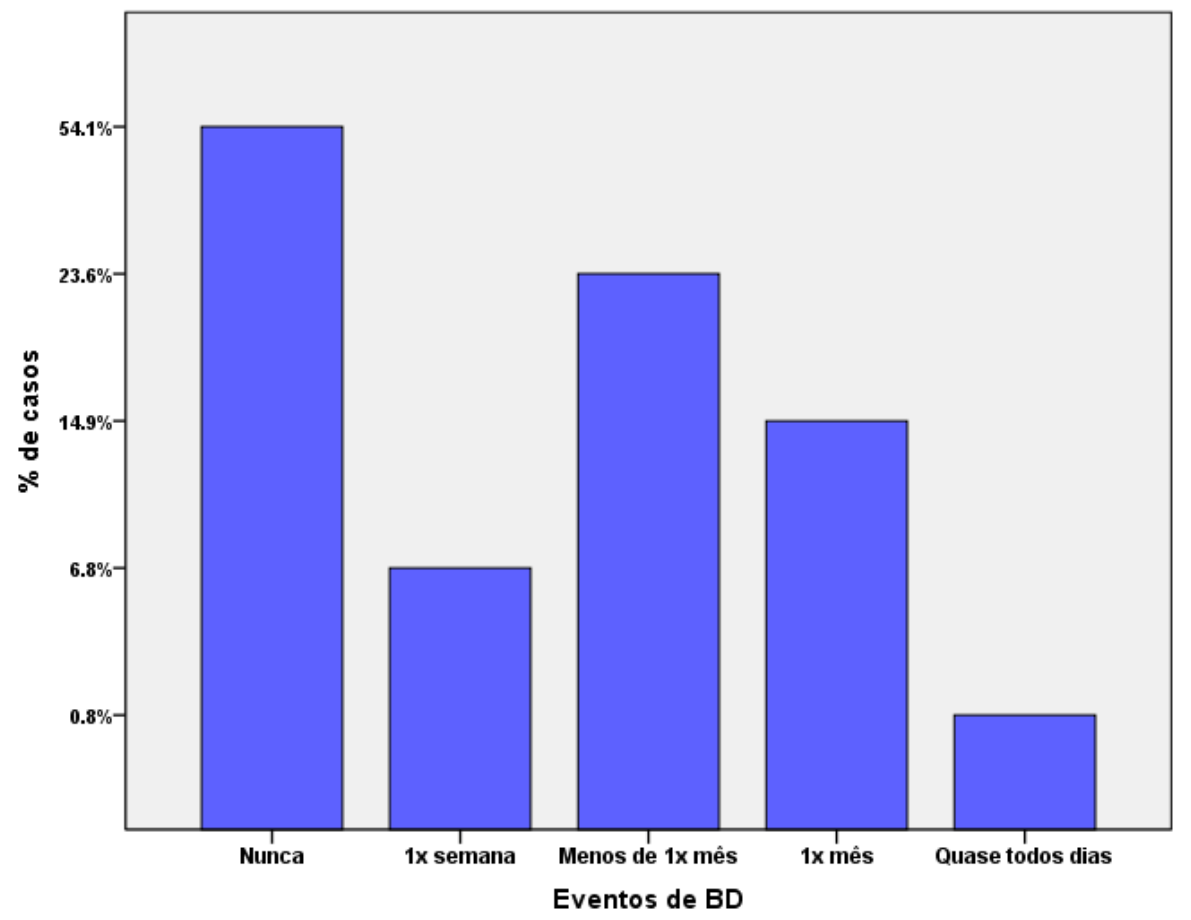

Gráfico 3. Frequência de Binge Drinking (Acima de 5 doses) entre os universitários.

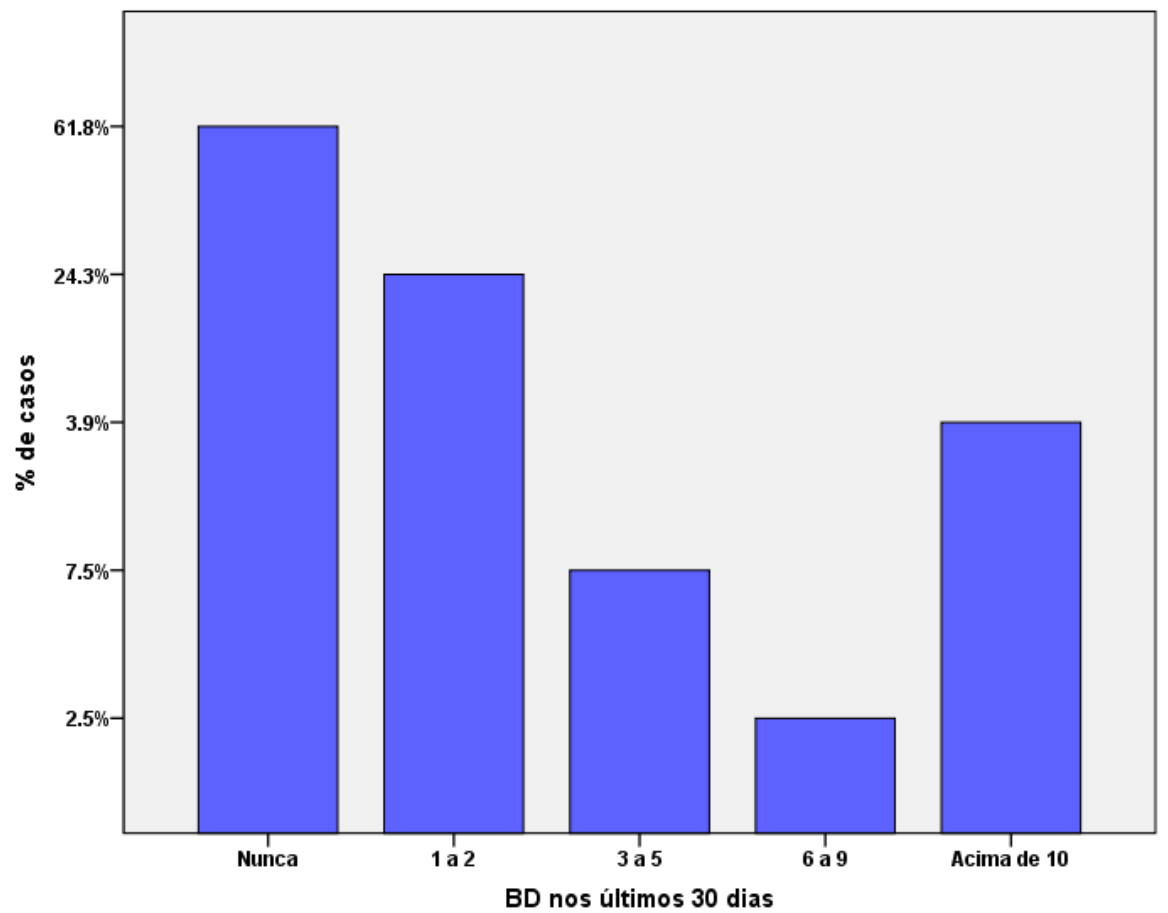

Gráfico 4. Prática de Binge Drinking (em dias) nos Últimos 30 dias. 
Em relação ao comportamento dos universitários na inter-relação do uso de álcool e drogas e comportamentos de risco (Gráfico 5), podemos destacar alguns dados preocupantes: 58,3\% ( $\mathrm{n}=302)$ universitários declararam que não usam preservativos nas relações sexuais, 13,7\% ( $n=71)$ tinham ingerido bebida alcoólica antes da relação sexual e 53,1\% ( $n=275)$ já tiveram mais de três parceiros sexuais, esses dados corroboram com dados da literatura, onde destacam que 0 uso de substâncias psicoativas principalmente $o$ álcool estão diretamente relacionadas com um aumento na probabilidade de comportamentos de risco, como podemos destacar o uso de camisinha e número de parceiros sexuais (WAGNER; ANDRADE, 2008) e que há uma relação direta entre o uso de drogas e álcool com o aumento do risco de contrair de doenças sexualmente transmissíveis e HIV (PILLON et al., 2005). Também podemos destacar que 32,8\% ( $n=170)$ utilizam apenas métodos contraceptivos orais e injetáveis, o que nos remete que, há apenas uma preocupação com o risco de gravidez e não ao risco de contrair alguma doença sexualmente transmissível.

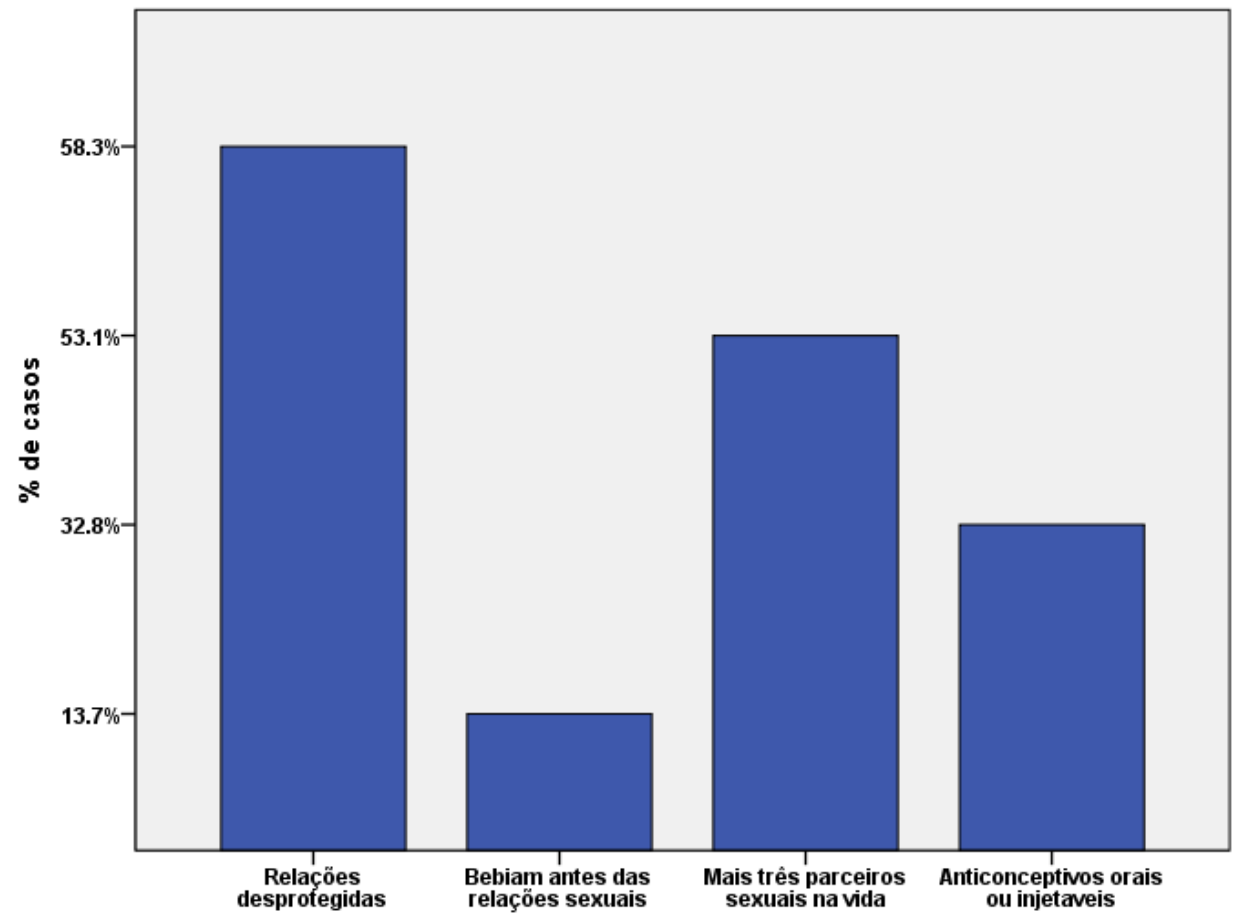

Gráfico 5. Perfil do universitário, quanto ao uso de álcool e drogas e comportamentos de risco. 
Em síntese, o sexo masculino apresentou altos índices de consumo em relação ao sexo feminino, porém quando comparados com o padrão de consumo ou $\mathrm{BD}$, as mulheres parecem ingerir maiores quantidades que os homens. Quando relacionando o uso de álcool com o comportamento de risco, o sexo feminino demonstra maiores frequências de atividades sexuais, com maior número de parceiros e menor índice de medidas contraceptivas, ou seja, poucas referiram o uso de preservativos pelos parceiros, sugerindo a relação do consumo de álcool com a transmissão de Doenças Sexualmente Transmissíveis (DST's).

O álcool consumido de maneira abusiva é um dos principais fatores para doenças cardiovasculares e aumenta o risco a situações agravantes como acidentes de trânsito, brigas e homicídios, representando a maior causa de morte entre jovens, além ser considerada fator de predisposição para o consumo de outras drogas como tabaco, drogas ilegais e a manifestação de desordens psíquicas, como quadros de depressão e ansiedade (ANDRADE et al., 2010; MALTA et al., 2011).

Diferentes estudos evidenciam a alta prevalência do consumo de substâncias psicoativas, principalmente por adolescentes e jovens no ensino superior, que vivem um período de grandes alterações fisiológicas e comportamentais, com maior vulnerabilidade pela busca por sensações que podem estar associadas ao consumo de álcool, drogas e outras substâncias psicoativas, lícitas ou ilícitas. As informações sobre a relação entre o uso de drogas e comportamento de risco são necessárias para identificar estratégias e propiciar mudanças saudáveis no estilo de vida dos universitários no contexto do consumo de álcool, drogas e outras substâncias psicoativas (PILLON et al., 2005; CARDOSO et al., 2009; TSUDA; CHRISTOFF, 2017; LOUREIRO, 2012; REIS; OLIVEIRA, 2015).

A adolescência se caracterizar principalmente como uma fase de mudanças físicas, psíquicas e emocionais, dessa forma surge a curiosidade, os questionamentos, a vontade de conhecer e experimentar o novo, não se preocupando com os riscos e aumentando sua capacidade de 
independência, facilitando o primeiro contato com as drogas e outras substâncias psicoativas (LOUREIRO, 2012), além de ser uma fase de maior vulnerabilidade pelo momento vivenciado no ensino superior (FACHINI, 2013), um período inclusive marcado por festas que proporcionam momentos de maior interação social, distração e relaxamento (LOUREIRO, 2012; PILLON; CORRADI-WEBSTER, 2006).

O álcool é um psicoestimulante do SNC e, dentre seus efeitos, podemos destacar alterações que causam sensações de prazer, dessa forma sendo um atrativo para o consumido pelos jovens. Além do mais, é notável o crescimento exponencialmente no consumo de álcool, chegando à estimativa de dependência em cerca de 10 a $12 \%$ da população mundial e $11 \%$ da população brasileira (MENDONÇA et al., 2018).

\section{Conclusões}

O presente estudo indica a relação entre o consumo excessivo de bebidas alcoólicas e desenvolvimento de comportamentos de risco na população acadêmica de ensino superior consultada. O álcool é a substância psicoativa que apresentou os maiores percentuais de consumo da amostra total, sendo hipotético que esse resultado tenha relação com as mudanças físicas, psíquicas e emocionais dos jovens e em razão de ser uma substância lícita e desobrigada de prescrição (como medicamentos), portanto de fácil acesso. Há necessidade de novos estudos para a melhor compreensão das expectativas do padrão da amostra avaliada e para auxiliar na prevenção dos fatores de risco. Este estudo é sugerido como norte para a realização de novas pesquisas com maior amostragem representativa da população de acadêmicos jovens do ensino superior. 


\section{Referências}

ANDRADE, Arthur Guerra de; DUARTE Paulina do Carmo Arruda Vieira; OLIVEIRA, Lúcio Garcia de. I Levantamento nacional sobre o uso de álcool, tabaco e outras drogas entre universitários das 27 capitais brasileiras. Brasília: Secretaria Nacional de Políticas sobre Drogas SENAD/FMUSP; $2010.282 \quad$ p. Disponível em: <http://admin.cisa.org.br/userfiles/ILevantamentoNacionalUniversitario.pdf>. Acessado em 11 abril 2021.

BOCHNER, Rosany. Perfil das intoxicações em adolescentes no Brasil no período de 1999 a 2001. Caderno de Saúde Pública, v. 22, n. 3, p. 587-95, 2006. http://dx.doi.org/10.1590/S0102-311X2006000300014

BOYLE, Peter et al. Alcohol: Science, Policy and Public Health. Oxford: Oxford University Press; 2013. 448 p.

CARDOSO, Higor Chagas et al. Avaliação da qualidade do sono em estudantes de medicina. Revista Brasileira de Educação Médica., v. 33, n. 3, p. 349-55, 2009. https://doi.org/10.1590/S0100-55022009000300005

CARDOSO, Fernanda Mourão et al. Factors associated with practice of binge drinking among students of health. Revista CEFAC, v. 17, n. 2, p. 475484, 2015. https://doi.org/10.1590/1982-021620158914

CENTRO BRASILEIRO DE INFORMAÇÕES SOBRE DROGAS PSICOTRÓPICAS (CEBRID). Levantamento sobre o consumo de substâncias psicoativas entre estudantes de ensino fundamental $\left(8^{\circ} \mathrm{e}\right.$ 9o ano) e médio (1 a 3 ono) da rede particular do município de São Paulo, n. 66, 2010.

FACHINI, Alexandre; FURTADO, Erikson Felipe. Alcohol use and drinking expectations among college students: an analysis of sex differences. Psicologia: Teoria e Pesquisa, v. 29, n. 4, p. 421-428, 2013. https://doi.org/10.1590/S0102-37722013000400008

JOHNSTON, Lloyd D. Monitoring the Future: National results on adolescent drug use: Overview of key findings, 2006. Bethesda: National Institute on Drug Abuse, 2007. 76 p.

LARANJEIRA, Ronaldo et al. I levantamento nacional sobre os padrões de consumo de álcool na população brasileira, Brasília, 2007. Disponível em:

$<$ http://bvsms.saude.gov.br/bvs/publicacoes/relatorio_padroes_consumo_alc ool.pdf>. Acessado em 11 abril 2021.

LOUREIRO, Liliana Almeida. Consumo de substâncias psicoativas e estilos de vida nos estudantes do ensino superior. Dissertação (Mestrado em Enfermagem Comunitária) - Escola Superior de Enfermagem de Coimbra: Escola Superior de Enfermagem de Coimbra, p. 121, 2012. 
MALTA, Deborah Carvalho et al. Prevalência do consumo de álcool e drogas entre adolescentes: análise dos dados da Pesquisa Nacional de Saúde Escolar. Revista brasileira de Epidemiologia. São Paulo, v. 14, supl. 1, p. 136 -146 , setembro. 2011.

MENDONCA, Ana Karina Rocha Hora; JESUS, Carla Viviane Freitas de; LIMA, Sonia Oliveira. Fatores Associados ao Consumo Alcoólico de Risco entre Universitários da Área da Saúde. Revista Brasileira de Educação Médica, v. 42, n. 1, p. 207-215, 2018. https://doi.org/10.1590/1981$\underline{52712018 v 42 n 1 r b 20170096}$

NUNES, Jaceilde Mendes et al. Consumo de bebidas alcoólicas e prática do binge drinking entre acadêmicos da área da saúde. Revista de Psiquiatria Clínica. v. 39, n.3, p. 94-99, 2012. https://doi.org/10.1590/S0101$\underline{60832012000300005}$

Rocha, Leandro Augusto et al. Alcohol use by medical students in Minas Gerais State, Brazil. Revista Brasileira de Educação Médica, v. 35, n. 3, p. 369-375, 2011. https://doi.org/10.1590/S0100-55022011000300010

PECHANSKY, Flavio; SZOBOT, Claudia Maciel; SCIVOLETTO, Sandra. Uso de álcool entre adolescentes: conceitos, características epidemiológicas e fatores etiopatogênicos. Revista Brasileira de Psiquiatria, São Paulo, v. 26 (Supl.), p.14-17, 2004. https://doi.org/10.1590/S1516-44462004000500005

PILLON, Sandra Cristina, O'brien, Beverley; CHAVEZ, Ketty Aracely Piedra. The relationship between drugs use and risk behaviors in Brazilian university students. Revista Latino-Americana de Enfermagem, v. 13, n. SPE2, p. 1169-1176, 2005. https://doi.org/10.1590/S0104-11692005000800011

PILLON, Sandra Cristina; CORRADI-WEBSTER, Clarissa Mendonça. Teste de identificação de problemas relacionados ao uso de álcool entre estudantes universitários. Revista de Enfermagem UERJ, v. 14, n. 3, p. 325-32, 2006.

PUIG-NOLASCO, Angel; CORTAZA-RAMIREZ, Leticia; PILLON, Sandra Cristina. Consumo de alcohol entre estudiantes mexicanos de medicina. Revista Latino-Americana de Enfermagem, v. 19, p. 714-21, 2011. https://doi.org/10.1590/S0104-11692011000700008

REIS, Tatiana Gonçalves dos; OLIVEIRA, Luiz Carlos Marques de. Pattern of alcohol consumption and associated factors among adolescents students of public schools in an inner city in Brazil. Revista Brasileira de Epidemiologia, v. 18, n. 1, p. 13-24, 2015. https://doi.org/10.1590/1980$\underline{5497201500010002}$

STRAUCH, Eliane Schneider et al. Uso de álcool por adolescentes: estudo de base populacional. Revista de Saúde Pública, v. 43, n. 4, p. 647-655, 2009. https://doi.org/10.1590/S0034-89102009005000044

SANCHEZ, Zila M.; RIBEIRO, Karen J.; WAGNER, Gabriela A. Binge Drinking Associations with Patrons' Risk Behaviors and Alcohol Effects after 
Leaving a Nightclub: Sex Differences in the" Balada com Ciência" Portal Survey Study in Brazil. PLoS One, v. 10, n. 8, e0133646, 2015. 10.1371/journal.pone.0133646

TAVARES, Beatriz Franck; BÉRIA, Jorge Umberto; LIMA, Mauricio Silva de. Prevalência do uso de drogas e desempenho escolar entre adolescentes. Revista de Saúde Pública, v. 38, p. 787-796, 2001. https://doi.org/10.1590/S0034-89102001000200008

TSUDA, Cristiane Armstrong; CHRISTOFF, Adriana de Oliveira. Avaliação do padrão de uso de estimulantes em uma faculdade de Curitiba-PR. Cadernos da Escola de Saúde, v. 1, n. 13, 2017.

TAVOLACCI, Marie-Pierre et al. Prevalence of binge drinking and associated behaviours among 3286 college students in France. BMC Public Health, v. 16, p. 178, 2016.

WAGNER, Gabriela Arantes; ANDRADE, Arthur Guerra de. Uso de álcool, tabaco e outras drogas entre estudantes universitários brasileiros. Revista de Psiquiatria Clínica, v. 35, Suppl 1, p. 48-54, 2008. https://doi.org/10.1590/S0101-60832008000700011

WORLD HEALTH ORGANIZATION (WHO). Global strategy to reduce the harmful use of alcohol. Geneva, 373p, 2010.

WORLD HEALTH ORGANIZATION (WHO). Global status report on alcohol and health. Geneva, 390p, 2014. 